\title{
娄Sociología
}

Sánchez-Salcedo, J. F., Arboleda, M. A., Montes, M., Castrillón, M. C., Ríos, S. V., \& Soto, A. (2018). Perfil social y económico de los pacientes diagnosticados con esquizofrenia en Cali. Revista de Sociología 33(1), 58-76. doi: 10.535/0719-529X.2018.51850

\section{Perfil social y económico de los pacientes diagnosticados con esquizofrenia en Cali}

\author{
[Social and economic profile of patients diagnosed with schizophrenia in Cali] \\ José Fernando Sánchez-Salcedo1(i), María Adelaida Arboleda1 (iD, Mateo Montes (D), \\ María del Carmen Castrillón 1 (D), Sandra Viviana Ríos ${ }^{2}$ (D), \& Angélica Soto² (D) \\ ${ }^{1}$ Universidad del Valle, ${ }^{2}$ Hospital Departamental Psiquiátrico Universitario del Valle
}

\begin{abstract}
Resumen
El objetivo de este artículo consiste en la construcción de un perfil social, económico y demográfico de la población registrada con el Sistema de Selección de Beneficiarios para Programas Sociales (SISBEN) del Hospital Departamental Psiquiátrico Universitario del Valle (HDPUV) entre el 2009 y el 2017, cuyo diagnóstico se encuentra dentro del clúster de esquizofrenia, trastorno esquizotípico o trastorno de ideas delirantes. Se optó por un trabajo en el que se complementó la información entre las bases de datos del SISBEN de Cali y la del HDPUV para profundizar en datos sobre pobreza y vulnerabilidad de las personas que son potenciales beneficiarios de programas sociales estatales. Finalmente, el documento sugiere que hay una necesidad por estudiar la relación entre la pobreza, vulnerabilidad y salud mental, que no es casual, pero sí implica una prolongación o recrudecimiento de un ciclo de pobreza y de dificultades para la atención de los trastornos mentales agudos.
\end{abstract}

Palabras clave: salud mental, enfermedad mental, pobreza, vulnerabilidad, esquizofrenia.

\begin{abstract}
The objective of the present article is to construct a social, economic and demographic profile of the population registered with the System for the Identification of Potential Social Programme Beneficiaries (SISBEN) at the Hospital Departamental Psiquiátrico Universitario del Valle (HDPUV) in Cali, Colombia between 2009 and 2017, whose diagnosis falls within the cluster of schizophrenia, schizotypal personality disorder, or delusional disorder. Data from the SISBEN and HDPUV databases were compared in order to explore the level of poverty and vulnerability of those individuals who are considered potential beneficiaries of state-run social programmes. Finally, the paper proposes the need for further study of the relationship between poverty, vulnerability and mental health which, far from being coincidental, implies a prolongation or exacerbation of the cycle of poverty and of challenges relating to the treatment of acute mental disorders.
\end{abstract}

Keywords: mental health, mental illness, poverty, vulnerability, schizophrenia.

Contacto: La comunicación sobre este artículo debe ser enviada a José Fernando Sánchez-Salcedo, email: jose. sanchez@correounivalle.edu.co

Financiamiento: Esta investigación fue financiada por la Convocatoria de apoyo a Centros Institutos de investigación- CI 147-51209517 de la Vicerrectoría de investigaciones de la Universidad del Valle, en el marco del convenio de cooperación entre la Universidad del Valle-Cidse y el Hospital Departamental Psiquiátrico Universitario del Valle. 


\section{INTRODUCCIÓN}

El presente trabajo se ha realizado con el objetivo de construir un perfil social y económico de la población registrada en el Sistema de Selección de Beneficiarios para Programas Sociales (SISBEN) de Cali que ha sido atendida en el Hospital Departamental Psiquiátrico Universitario del Valle (HDPUV) entre el 2009 y agosto de 2017, pero cuyo diagnóstico hace parte del clúster de esquizofrenia, trastorno esquizotípico y trastorno de ideas delirantes. La decisión de centrarse en una población específica de pacientes de este hospital permite corroborar información entre las bases de datos del centro y la del SISBEN y ahondar en la relación que existe entre sus trastornos mentales, sus características socioeconómicas, de tratamiento y atención.

Al hablar del clúster de esquizofrenia, trastorno esquizotípico y trastorno con ideas delirantes, hacemos referencia a las clasificaciones psiquiátricas del DSM (Diagnostic and Statistical Manual of Mental Disorders) de la Asociación Americana de Psiquiatría y a la CIE (Clasificación Internacional de Enfermedades) de la Organización Mundial de la Salud, ambas con sus diferentes versiones. Desde estas clasificaciones, se habla del espectro de enfermedades que tienen como característica central un compromiso para establecer los límites entre la experiencia interna de la mente y la realidad externa (Lieberman \& First, 2018). La diferencia principal en este sentido entre ambas clasificaciones está dada por el trastorno de personalidad esquizotípica, que el CIE10 enmarca en el espectro de la esquizofrenia (F20 a F29), en tanto que el DSM5 lo enlista en los trastornos de personalidad.

Las enfermedades del clúster en mención se distinguen unas de otras por tres diferentes características: (a) su duración (e.g., más de seis meses para esquizofrenia, menos de un mes para trastorno psicótico breve), (b) el perfil de síntomas (e.g., múltiples síntomas psicóticos como delirios, alucinaciones, comportamiento desorganizado en esquizofrenia y solo delirios en el trastorno de ideas delirantes), y (c) la relación con síntomas afectivos (e.g., su concurrencia con depresión o manía en el trastorno esquizoafectivo) (Lieberman \& First, 2018).

Es importante aclarar que el constructo "esquizofrenia" y las psicosis relacionadas, no se constituyen en entidades externas fijas, sus características y delimitaciones han variado a lo largo del tiempo, por lo tanto, los asumimos como cuadros heterogéneos, multidimensionales, con cambios importantes de manera longitudinal, sujeto a las influencias teóricas que el grupo de psiquiatras asistenciales tenga en el momento.

En la revisión de literatura realizada por McGrath, Saha, Welham, El Saadi1, MacCauley, y Chant (2004), la tasa promedio de incidencia para esquizofrenia fue de 15.2 por 100.000 habitantes en diferentes poblaciones a nivel mundial. La distribución por género mostró de manera consistente en diferentes estudios, una mayor presencia de este tipo de trastornos en población masculina, con una razón hombre es a mujer de 1.4 es a 1. También encontraron mayor prevalencia de este grupo de enfermedades en zonas urbanas, en comparación con la población rural. El inicio de los síntomas de este tipo de 
psicosis se produce en la adolescencia y adultez temprana, siendo más temprano en hombres. Estudios en Colombia han encontrado edades promedio de inicio de 24.5 años para hombres y 27.5 años para mujeres (Sánchez, Tellez \& Jaramillo, 2012), con un curso de mayor deterioro en hombres que en mujeres.

Castillo-Martell (2014) describió la presencia de deterioro significativo en aspectos clínicos, ocupacionales, educativos y relacionales en pacientes con un promedio de 10 años de enfermedad consultantes a un hospital mental de Lima, con percepción familiar negativa frente sus proyectos básicos de desarrollo familiar. Sumado a esto, las personas que presentan enfermedades mentales crónicas y graves como las del clúster de esquizofrenia, son objeto de rechazo y estigmatización, con creencias relacionadas a una potencial peligrosidad, situación que es peor cuando se trata de pacientes de género masculino (Boysen, Ebersole, Casner, \& Coston, 2014). Por otro lado, en un estudio piloto internacional sobre esquizofrenia mostró que existían diferencias significativas entre el pronóstico de los pacientes con esquizofrenia respecto a su procedencia, es decir, los países en desarrollo contaban con un pronóstico considerablemente mejor en relación con los países desarrollados o del Primer Mundo (Hopper \& Wanderling, 2000). Como parte de dicho estudio, en Cali se realizó un seguimiento de 26 años de duración en 101 pacientes del Hospital Psiquiátrico. Al finalizar los 26 años de seguimiento, la población tenía un promedio 48.8 años para hombres y 52 años para mujeres y había disminuido el número de solteros e incrementado el número de casados y separados. Los investigadores no observaron cambios en el estrato económico del sitio de vivienda, ni evidencia de deterioro en sus condiciones de vida; el $46 \%$ de los pacientes tuvo lo que los investigadores describieron como una valoración favorable en relación con el curso clínico longitudinal (León \& León, 1996).

Respecto a la asociación entre la situación social y económica con las enfermedades mentales, hay suficiente evidencia de la fuerte relación entre pobreza y problemas del espectro de la psicosis, en particular con la esquizofrenia, donde la pobreza es un factor desencadenante $y$ perpetuador de los síntomas. Diferentes estudios, incluyendo el estudio clásico de 1939 en Chicago, hasta estudios más recientes de la primera década del siglo XXI, muestran una relación entre el diagnóstico de esquizofrenia y la procedencia de barrios económicamente deprivados (Read, 2010). Desde mediados del siglo XX a la actualidad se ha estudiado la relación entre tipo de tratamiento y condición económica. Por ejemplo, Myers \& Bean (1968), describían que los pacientes en condición de pobreza recibían más tratamientos hospitalarios que los pacientes de clase media trabajadora, y el grupo de pacientes con vulnerabilidad económica recibía en mayor proporción tratamientos físicos (e.g., terapia electroconvulsiva, lobotomía). Abramowitz \& Dokecki (1977) reportaron que los pacientes de clase media recibían de manera preferencial farmacoterapia y psicoterapia (Read, 2010).

Así, el estudio de la salud mental y su relación con la pobreza se ha restringido a interpretaciones de indicadores de pobreza con connotaciones materiales, pero al ahondar en las condiciones de vulnerabilidad y en la noción del bienestar, se comprende que hay otros elementos más por abordar que complejizan este vínculo. Además, el impacto de las enfermedades 
mentales se extiende más allá de un diagnóstico o tratamiento clínico porque repercute en aspectos sociales, económicos o laborales, lo que implica que se profundicen las condiciones de vulnerabilidad de la persona.

\section{MÉTODO}

La estrategia metodológica utilizada para realizar la caracterización del perfil social y económico de pacientes diagnosticados con esquizofrenia del HDPUV supuso la consulta de la base de datos de historias clínicas del Hospital, la cual se cruzó con la base de datos del SISBEN. El uso de los datos sociales, económicos y demográficos provenientes del SISBEN se debe a que las personas registradas en esta base de datos son, en principio, las más vulnerables de la población colombiana. De este modo, el objetivo fue complementar la información consignada en las historias clínicas, las cuales se centran más en los aspectos médicos y de diagnóstico, dejando en un segundo plano los datos sobre los informantes. Para la realización del perfil se tuvieron en cuenta variables como sexo, estado civil, nivel educativo, información socioeconómica y datos relacionados con el hogar, así como indicadores como necesidades básicas insatisfechas, esto con el fin de determinar un vínculo posible entre los diagnósticos y la situación socioeconómica de los pacientes.

La población que ha sido estudiada en esta investigación consiste en aproximadamente un $20 \%$ de los pacientes registrados en SISBEN. Se busca mostrar su compleja condición de vulnerabilidad y las dificultades que deben afrontar en diversas esferas de su vida cotidiana, las que no se remiten solo a su salud mental, sino a elementos socioeconómicos, de atención, cuidado y demográficos. Hipotetizando que el contexto social, económico y político, que es propicio para que los índices de problemas de salud mental serían preocupantes en los sectores vulnerables y a su vez, para que las posibilidades de acceso a programas de atención en salud mental se limiten.

El SISBÉN es un sistema estatal colombiano diseñado para la identificación de la población en condición de vulnerabilidad, que tiene por finalidad distribuir el acceso a determinados programas sociales que los beneficiarios puedan requerir. Es un tipo de política pública desarrollada por el Estado colombiano para cerrar la brecha de la pobreza, promover el acceso al empleo digno, velar por la salud y la equidad. En ese sentido, el objetivo principal consiste en la generación de condiciones que permitan a todas las personas crear y proteger sus activos, además de desarrollarse a nivel personal y social.

Al momento de generación de esta investigación, se han implementado tres versiones del SISBEN, por lo cual se describen a continuación los aspectos más relevantes: (a) el SISBEN I se construyó con un énfasis en una metodología de carácter utilitarista del bienestar, considerando que las condiciones de acceso a bienes y servicios es central para comprender y clasificar la pobreza; (b) el SISBÉN II se propuso como una reestructuración tras los aprendizajes de su versión anterior, reconociendo en este caso la necesidad de construir un índice más y 
Sánchez-Salcedo, J. F., Arboleda, M. A., Montes, M., Castrillón, M. C., Ríos, S. V., \& Soto, A. (2018). Perfil social y económico de los pacientes diagnosticados con esquizofrenia en Cali. Revista de Sociología 33(1), 58-76. doi: 10.535/0719-529X.2018.51850 excluyendo las variables que se podían manipular con facilidad por parte de los hogares o personas, como el ingreso per cápita, equipamiento, entre otras variables, para ser sustituidas por otras más confiables y de fácil identificación para la persona que encuesta, como el material de los pisos, paredes o techos; (c) el SISBÉN III se diseña como un instrumento que reevalúa las ediciones previas y se focaliza en un índice de caracterización de las personas y los hogares vulnerables, para permitir la consolidación de la información respecto a la asignación de recursos para programas sociales, es decir, fue pensado como un método para abordar la pobreza desde una perspectiva multidimensional con el objetivo de minimizar los errores durante el proceso de recolección de los datos.

El HDPUV es un centro asistencial departamental, público y monovalente situado en la ciudad de Cali. Es una institución para la atención de personas con trastornos y problemas mentales agudos. Cuenta con 224 camas, de las cuales 65 son usadas para cuidado crítico y 159 para el cuidado intermedio psiquiátrico. El promedio de estancia hospitalaria es de 21 días. Por las características descritas, su ubicación estratégica en el suroccidente de Colombia, es el centro de mayor complejidad a nivel psiquiátrico y una entidad de referencia en asuntos de salud mental.

El HDPUV como la mayor parte de hospitales mentales públicos del mundo, ha albergado a población predominantemente de bajos recursos económicos. Inicialmente por su condición de institución de beneficencia y posteriormente desde la década de los noventa, bajo el modelo del sistema general de seguridad social de salud, tiene una contratación significativamente mayor con aseguradores del régimen subsidiado, población caracterizada a través del SISBEN como vulnerable.

Son 64,269 las personas registradas en la base de datos del HDPUV para el período estudiado. No todas las personas en este registro son pacientes, pues solo con solicitar una cita en la institución, los datos básicos de las personas ingresan en la base de datos. Para que una persona sea clasificada como paciente es necesario que se cree una historia (ficha) clínica a su nombre y por ende que haya sido atendida por uno de los profesionales de la entidad, quien tras finalizar la consulta registra el diagnóstico del paciente.

Tabla 1. Distribución de la población del HDPUV

\begin{tabular}{lcccccc}
\hline & \multicolumn{2}{c}{ Registros en HDPUV } & \multicolumn{2}{c}{ Pacientes de HDPUV } & \multicolumn{2}{c}{ Pacientes de HDPUV con } \\
& \multicolumn{2}{c}{ SISBÉN } \\
\cline { 2 - 7 } Población & $\mathrm{n}$ & $\%$ & $\mathrm{n}$ & $\%$ & $\mathrm{n}$ & $\%$ \\
\hline Hombres & 33,495 & 52.1 & 29,541 & 51.8 & 8,876 & 47.9 \\
Mujeres & 30,774 & 47.9 & 27,446 & 48.2 & 9,641 & 52.1 \\
Total & 64,269 & 100 & 56,987 & 100 & 18,517 & 100 \\
\hline
\end{tabular}


Sánchez-Salcedo, J. F., Arboleda, M. A., Montes, M., Castrillón, M. C., Ríos, S. V., \& Soto, A. (2018). Perfil social y económico de los pacientes diagnosticados con esquizofrenia en Cali. Revista de Sociología 33(1), 58-76. doi: 10.535/0719-529X.2018.51850
Como se aprecia en la Tabla 1, de los 64,269 registros, 56,897 son pacientes, de los cuales 18,517 estaban registrados en la base de datos del SISBEN III para la ciudad de Cali. El incremento en el número de mujeres al filtrar los datos de los pacientes con SISBEN, se explica porque el $54 \%$ de la población de Cali está identificada por el SISBEN, de los cuales el $53.5 \%$ son mujeres. Esta tendencia a un mayor registro de casos femeninos se da porque es un encuestador el que se dirige a los hogares para cumplimentar los formularios y esta condición coindice con que suelen ser más los casos de mujeres en disposición para atender al encuestador, además de su interés por hacer parte de programas sociales como Familias en Acción.

Durante la atención de un paciente en el HDPUV, profesionales de diversas especialidades participan del proceso y a su vez, al momento de atender a la persona, van a registrar un diagnóstico para la consulta. De esta manera, la historia clínica de un paciente reúne diferentes miradas, métodos y concepciones en torno a la enfermedad mental. Teniendo en cuenta lo anterior, se optó por filtrar tan solo los diagnósticos de las historias clínicas relacionadas con la atención en psiquiatría, siendo descartados entonces aquellos sin un vínculo directo con la práctica psiquiátrica, como puede ser la atención desde terapia ocupacional, por ejemplo.

A raíz de lo anterior, los tipos de historia clínica seleccionados para aplicar el filtro de diagnósticos de los pacientes fueron:

- Historia clínica psiquiátrica de primera vez.

- Historia clínica psiquiátrica de primera vez con examen físico.

- Historia clínica de ancianos.

- Seguimiento comunitario de ingreso.

- Atención domiciliaria (anexo de historia clínica de seguimiento).

- Evolución de historia clínica del hospital de día.

- Evolución de historia clínica de hospitalización.

- Evolución de historia clínica por consulta externa.

- Evolución de ingreso por hospital de día.

- Nota de urgencias psiquiátricas.

- Seguimiento comunitario evolución.

El proceso de filtrado descrito implicó que de los 18,517 pacientes de la Tabla 1, la cifra se redujera a 17,650. Finalmente, se presenta el último diagnóstico asignado, considerando que es ese último diagnóstico psiquiátrico el que da cuenta de la trayectoria de atención del paciente. 


\section{RESULTADOS}

\section{Diagnósticos}

En la Figura 1 se presenta la información de los diagnósticos agrupados por clúster, donde se

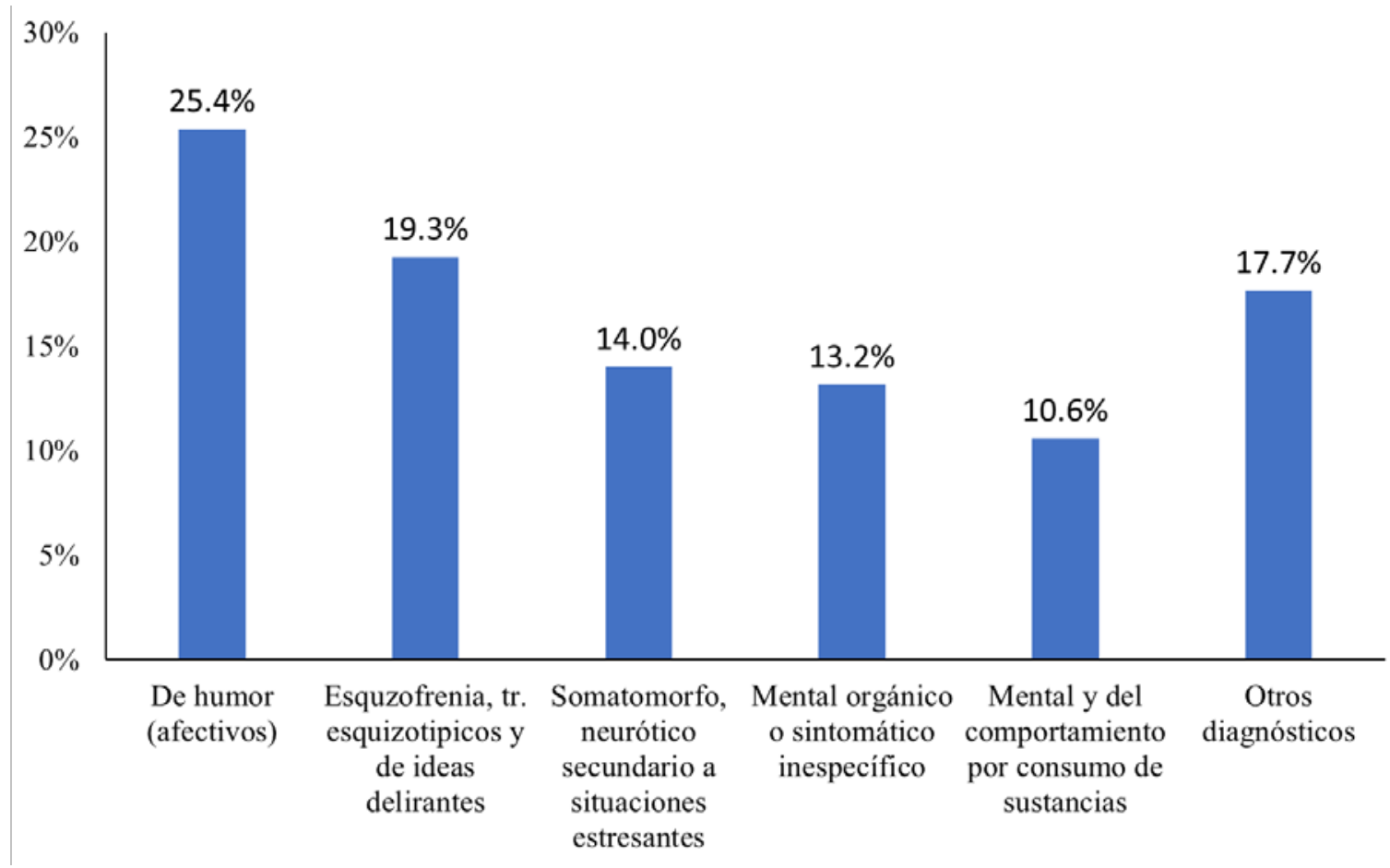

Figura 1. Tipos de trastornos diagnosticados en pacientes con registro en el SISBÉN.

Los trastornos psiquiátricos se caracterizan por ser catalogados como parte de las enfermedades que ocasionan una pérdida mayor de años de vida, incrementan la consulta en los servicios de salud, los costos asociados con su atención son altos y la calidad de vida de quienes son diagnosticados con ellos se deteriora. Los trastornos mentales son frecuentes en todo el planeta y se estima que su prevalencia en adultos es del 12 a $48 \%$ a lo largo de la vida y, para los últimos 12 meses entre el 8 al $29 \%$ (World Health Organization [WHO], 2008). En encuentra que son los trastornos del humor los más frecuentes, seguidos por la esquizofrenia, trastorno esquizotípico y trastornos de ideas delirantes. 
bipolares, sin embargo, el hallazgo más llamativo está constituido por el segundo grupo diagnóstico, que corresponde al clúster de la esquizofrenia y las psicosis asociadas

Según estudios previos, la incidencia promedio de la esquizofrenia se estima en 15.2 casos por cada 100,000 habitantes (McGrath et al., 2004), con una prevalencia de entre 4 a 8 personas por cada 1,000 habitantes (McGrath, Saha, Chant, \& Welham, 2008; Saha, Chant, Welham, \& McGrath, 2005). En estudios como el de Jablensky (2000) la prevalencia a lo largo de la vida es del $1 \%$, mientras que en una mirada anual es del 1.4 al $4.6 \%$ por cada 1,000 habitantes y una incidencia entre 0.16 a 0.42 casos por 1,000 habitantes. Es importante resaltar sobre la incidencia de este diagnóstico que pese a ser calculada en la mayor parte de la literatura para la población global a lo largo de la vida entre un 1\% o $2 \%$, en el HDPUV el clúster que la integra le corresponde casi un $20 \%$ del total de diagnósticos (ver Figura 1). La interpretación de este resultado pasa por considerar que el estudio se realizó con población consultante a un hospital público que es centro de referencia para otras instituciones del municipio de Cali y territorios circundantes. No fue objeto de este trabajo realizar la comparación con población rural, sin embargo, como se ha descrito en la literatura, otro elemento para tener en cuenta es la mayor prevalencia de psicosis del clúster de esquizofrenia en población urbana (McGrath et al., 2004)

Entre los pacientes diagnosticados dentro del clúster esquizofrenia, trastorno esquizotípico o trastorno de ideas delirantes, el 60\% de ellos son hombres. Al respecto y de acuerdo con la revisión sistemática realizada por Aleman, Kahn, \& Selten (2003), los documentos recientes que se han publicado muestran que, aparentemente, el riesgo de ser diagnosticados con esquizofrenia es mayor para los hombres. De todos modos, la posibilidad que la esquizofrenia sea menos reconocida en mujeres aún no ha sido estudiada en profundidad, aunque los autores sí resaltan que hay varias vías para explicar esta cifra: la incidencia de la esquizofrenia es idéntica para ambos sexos, pero se detecta con mayor facilidad en los hombres porque la enfermedad se manifiesta en formas más severas y la existencia de factores adicionales que pueden hacer que la psicosis en mujeres tenga menos probabilidades de ser atendida por profesionales para asignar el tratamiento correspondiente, o que los síntomas sean reconocidos; y una segunda posibilidad es que la incidencia real sea mayor para los hombres, porque la gravedad del trastorno complica la interpretación. En conclusión, los datos muestran que ser hombre es un factor que puede ser considerado como un riesgo mayor para el desarrollo de una forma más severa de esquizofrenia y a su vez, más fácilmente reconocible.

Distinciones por variables sociales y económicas

La pirámide poblacional (ver Figura 2) muestra que hay una concentración de pacientes entre los 20 y 34 años, la cual suma en total el $40 \%$ de los casos, cifra que se distribuye en un $30 \%$ para los hombres y un 10\% para las mujeres. En relación con las mujeres, el porcentaje empieza a hacerse más alto entre los 40 a los 59 años, grupo de edad al que le corresponde un $18 \%$ de los casos. Esto muestra que los hombres jóvenes son más diagnostican dentro de este clúster, mientras que para las mujeres su porcentaje crece a partir de los 40 años hasta los 59 e incluso supera al de 
los hombres. Ahora bien, hay que destacar que la población de pacientes del HDPUV en otras investigaciones que se han realizado, han mostrado que los jóvenes tienden a ser hombres, mientras que en la población más vieja predominan las mujeres.

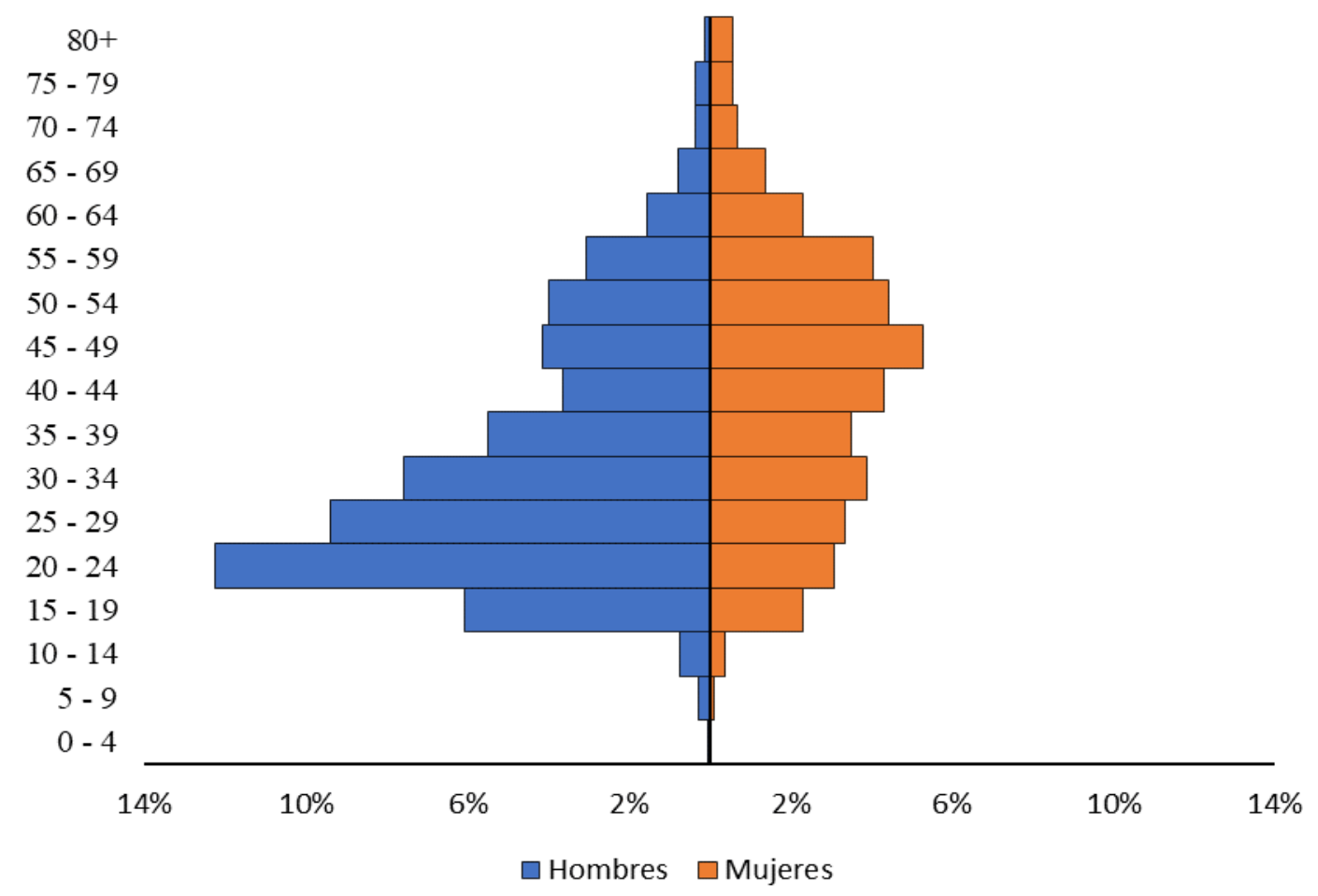

Figura 2. Pirámide poblacional de los pacientes.

En la literatura se describe una tasa de prevalencia similar para hombres y mujeres, discretamente mayor en hombres, (McGrath et al., 2004; Sánchez, Tellez y Jaramillo, 2012; Alemán, Kahn, \& Selten, 2003), de manera que el predominio masculino para este clúster de esquizofrenia, en particular en jóvenes de una nuestra población hospitalaria, puede tener explicaciones relacionadas a sesgos y estereotipos negativos asociados a este grupo de enfermedades, tal como lo propone Boysen y colaboradores (2014), por otro lado, en el metaanálisis realizado por Alemán y colaboradores
(2003) se menciona que una posibilidad de fallas en el diagnóstico del clúster de esquizofrenia lleva a reconocerlo más fácilmente en hombres jóvenes, e incluso sobre diagnosticarlo, para luego ser cambiado a lo largo del tiempo a otro espectro de enfermedades, por ejemplo trastornos afectivos bipolares o cuadros psicóticos asociados al uso de sustancias psicoactivas, en tanto que en las mujeres, hay mayor tendencia a identificar síntomas afectivos. Otra hipótesis en la diferencia de diagnóstico entre géneros y edades, se relaciona a la mención de un mayor riesgo de mortalidad en hombres 
con esquizofrenia frente a las mujeres (dos veces mayor).

Al desagregar los pacientes por estado civil o de pareja, el grupo mayoritario son los solteros (75.5\%) con tres cuartas partes de la población, seguido por los casados o en unión libre (13.9\%) y los separados o divorciados (7.9\%), siendo los viudos el grupo minoritario (2.7\%). Esto muestra que la vida en pareja al momento de realizar la encuesta o previo a ella es la que menos casos registra entre los pacientes y que las personas con este tipo de diagnósticos son en su mayoría solteras. Al desagregar el estado civil por sexo (ver Figura 3), la diferencia más significativa se encuentra en el grupo de quienes viven en pareja o lo han hecho en el pasado, es decir, son un $92.3 \%$ de mujeres entre los viudos, respecto a los separados o divorciados a las mujeres les corresponde un $67 \%$ de los casos y entre los casados o en unión libre, la población femenina es del 55.3\%. En cuanto a los hombres, predominan entre los solteros con el $67.3 \%$.

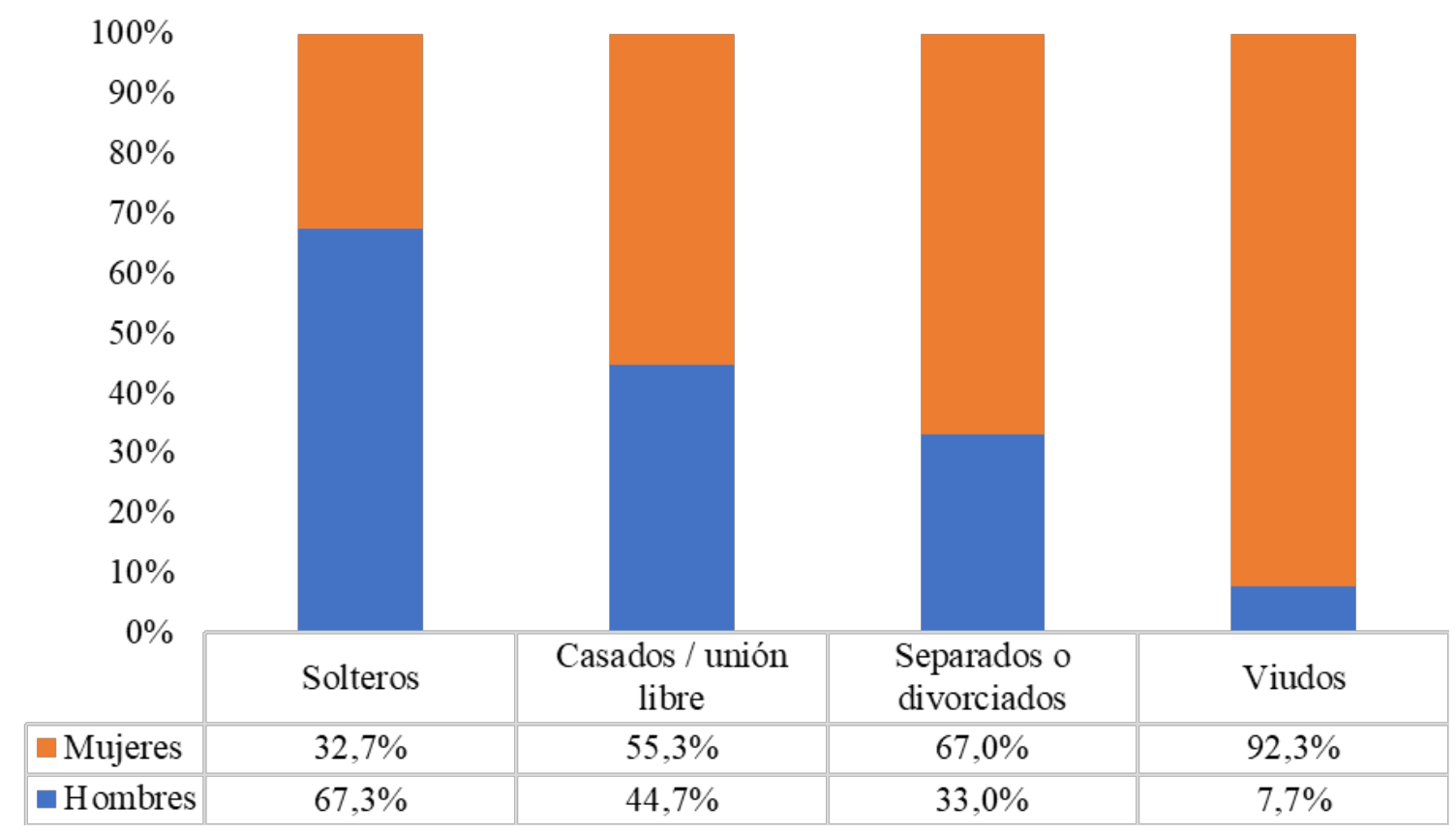

Figura 3. Pacientes por estado civil y por sexo

El inicio más temprano de los síntomas en hombres puede ser uno de los factores que expliquen la ausencia de establecimiento de relaciones estables en este grupo. Por otro lado, la población masculina que constituye la muestra presenta predominio de hombres jóvenes, en tanto que la femenina está representada por mujeres entre la cuarta y la sexta década de la vida, con posibilidad de haber establecido relaciones de pareja; hallazgo que es similar a lo encontrado en otras investigaciones (Salokangas, Honkonen, Stengard, \& Koivisto., 2001). Serían necesarios estudios complementarios para establecer otros determinantes relacionados a este hallazgo, teniendo en cuenta que el estudio longitudinal de esquizofrenia realizado en Cali (León \& León, 1996) mostró que, a largo plazo, las condiciones 
maritales variaban en la población masculina y las expectativas de construcción de relación y de actividad ocupacional eran considerablemente mejores a las observadas en ciudades europeas. El nivel educativo modal para los pacientes es secundaria $(52.5 \%)$, seguido de la primaria (36.9\%). La educación superior, la cual agrupa los estudios técnicos, tecnológicos, profesionales o de posgrado muestra tan solo un $2.6 \%$ de los casos, mientras que quienes presentan un nivel educativo nulo en términos de acceso a educación formal es del 8\%. De este modo, se identifica entonces que esta es una población en la que el nivel educativo se concentra en la formación de secundaria o primaria en alrededor de un $90 \%$, siendo este un dato significativo si se piensa que alrededor de tres de cada 100 pacientes se han formado en algún nivel de educación superior, siendo este un factor que complejiza las posibilidades de cerrar las brechas de vulnerabilidad y de acceso a mejores condiciones laborales o de calidad de vida.

$\mathrm{Al}$ analizar los datos por edad, se observó que los pacientes sin una actividad son el 43.4\%, seguidos por quienes trabajan (19.2\%) o se dedican a los oficios del hogar (15.6\%). Un 11.2\% está buscando trabajo, el 7.2\% estudia y el 3.5\% tiene alguna otra actividad. A modo de comparación, en un estudio en Australia se encontró que, entre pacientes con esquizofrenia, solo el $14.5 \%$ de los hombres y el $17 \%$ de las mujeres contaban con alguna clase de empleo. A su vez, únicamente el $25.4 \%$ de las mujeres y el 29.5\% de los hombres tuvieron un trabajo regular el año anterior a la investigación. (Carr, Neil, Halpin, Holmes, \& Lewin, 2003).

Las condiciones de vulnerabilidad, en conjunto con la situación de salud mental, son dos variables que pueden explicar las dificultades de esta población para acceder a un empleo o a una institución educativa. Aproximadamente el 20\% de los pacientes trabajan o se dedican a una actividad remunerada, siendo esta otra condición que profundiza su compleja condición socioeconómica; y un $11.2 \%$ busca trabajo, así que una décima parte de la población declaró en la encuesta que pretende acceder al mercado laboral, lo que conlleva a pensar al recordar la distribución de los grupos de edad en la pirámide poblacional (ver Figura 2) que el grueso de los pacientes se encuentra en edad de trabajar y por ende la cantidad es mínima en comparación con quienes no tienen una actividad principal definida, en otras palabras, de nuevo vale la pena considerar el planteamiento sobre las condiciones de vulnerabilidad y de salud mental que se erigen como una barrera de acceso al mercado laboral o la educación formal.

Los estratos socioeconómicos más bajos (i.e., 1, 2 y 3) representan el $99.5 \%$ de la población estudiada; los estratos altos (i.e., 4, 5 y 6) son solo el $0.5 \%$. A partir de estos datos se comprende que la población se concentra en los estratos bajobajo (38.7\%), bajo (39.7\%) y medio-bajo (18.5\%). Es decir, son pacientes que habitan viviendas clasificadas como de menores recursos y que son beneficiarias de subsidios en sus servicios públicos domiciliarios, a diferencia de lo que sucede con los estratos 5 y 6 que pagan sobrecostos por ser usuarios con recursos económicos mayores o el estrato 4 que ni paga sobrecostos, ni se beneficia de los subsidios.

El número de personas por hogar o tamaño del hogar, es una variable que dificulta el cuidado de un paciente con una enfermedad mental, dificultad que aumenta si se trata de una población diagnosticada con esquizofrenia, trastorno esquizotípico o trastorno de ideas 
delirantes porque son pacientes que precisan de condiciones de estabilidad y certidumbres en su rutina diaria para encaminar su atención, factor que se puede ver alterado en la medida en que el tamaño del hogar sea mayor, considerando no solo la cantidad de personas que lo habitan, sino aspectos como la convivencia o la distribución y uso de los espacios de la vivienda. En este caso, los pacientes con un diagnóstico que hace parte del clúster estudiado muestran una alta condición de vulnerabilidad, pues en cerca de un $22 \%$ de los hogares viven 6 personas o más y en alrededor de un $30 \%$ lo hacen 4 o 5 personas. Por otro lado, el $10.2 \%$ de pacientes que habitan hogares unipersonales, porque además del diagnóstico en salud mental que este documento aborda, su red de apoyo en el hogar es limitada y por ende su soporte en términos de cuidado y tratamiento es frágil.

Aproximadamente 7 de cada 10 pacientes (68.5\%) está afiliado al régimen de aseguramiento en salud en cualquiera de sus modalidades, lo que significa que la parte restante (31.5\%) de la población debe enfrentarse a unas condiciones de vulnerabilidad que no solo están atravesadas por factores sociales y económicos, sino de acceso a un proceso de cuidado en salud mental integral.

Por parte de quienes están afiliados, es el régimen subsidiado el que concentra la mayoría de los casos (80.6\%). Este es un dato que en parte se explica porque uno de los programas sociales más sólidos del SISBEN es el de la afiliación de la población al sistema de salud en el régimen subsidiado, así que es de esperarse que la proporción sea alta. Ahora bien, la anterior explicación no anula que el dato sea alto y sea una muestra más de las condiciones de vulnerabilidad de la población, que en este caso en particular son atendidas por medio de un programa social.

La pobreza monetaria se define como todo ingreso per cápita por debajo de la línea de la pobreza, la cual se comprende como el costo per cápita mínimo de la canasta básica de bienes alimentarios y no alimentarios, y que a su vez depende de la zona geográfica. $\mathrm{Al}$ respecto, en el 2016 el cálculo de la línea de la pobreza para el total nacional fue de $\$ 241,673$, mientras para Cali y su área metropolitana fue de $\$ 265,559$, y para los centros poblados y rural disperso fue de $\$ 159,543$. Por otro lado, la pobreza monetaria extrema es todo ingreso per cápita inferior a la línea de pobreza extrema, entendida como el costo per cápita mínimo de una canasta alimentaria que garantiza únicamente las necesidades básicas calóricas y que a su vez tiene en cuenta el área geográfica. En el año 2016, el total nacional fue de $\$ 114,692$, mientras para Cali y su área metropolitana fue de $\$ 119,957$ y para los centros poblados y rural disperso de $\$ 97,867 \quad$ (Departamento Administrativo Nacional de Estadística [DANE], 2017).

De acuerdo con el indicador de pobreza monetaria, el $90.7 \%$ de los pacientes viven por debajo de la línea de pobreza y el $61.7 \%$ de ellos lo hacen por debajo de la línea de pobreza extrema. En otras palabras, es una variable más que da cuenta de las agudas condiciones de vulnerabilidad, aunque desde una perspectiva económico. El anterior punto es clave, porque estos son datos que tienden a presentar incongruencias: primero por el modo en que se obtiene la información al ser una pregunta planteada por un encuestador del SISBEN con quien la relación de confianza para consultar por asuntos como el ingreso es mínima; por el posible interés de los encuestados por modificar los datos 
para ser registrados como más pobres en las bases de datos y así ampliar el margen de posibilidades para ser considerados como beneficiarios potenciales de los programas sociales del Estado; y finalmente porque es un indicador que se piensa la pobreza tan solo a partir del ingreso per cápita, lo que implica que se omite su perspectiva multidimensional.

Las necesidades insatisfechas (ver Figura 4) predominantes son el hacinamiento crítico y la dependencia económica. El hacinamiento crítico es un indicador que los recursos de la vivienda en la que habitan, al igual que el grupo de personas restantes, están siendo ocupados de un modo crítico. Para la construcción de este índice se incluyen a las viviendas que registran a más de 3 personas por habitación. Esta es una necesidad básica insatisfecha que corresponde con los datos observados de tamaño del hogar, es decir, las dificultades son claras para esta población y se reafirma la idea que, desde la óptica del cuidado en salud mental, para quien es diagnosticado dentro del clúster aquí abordado, son demasiado complejas para su tratamiento y estabilidad.

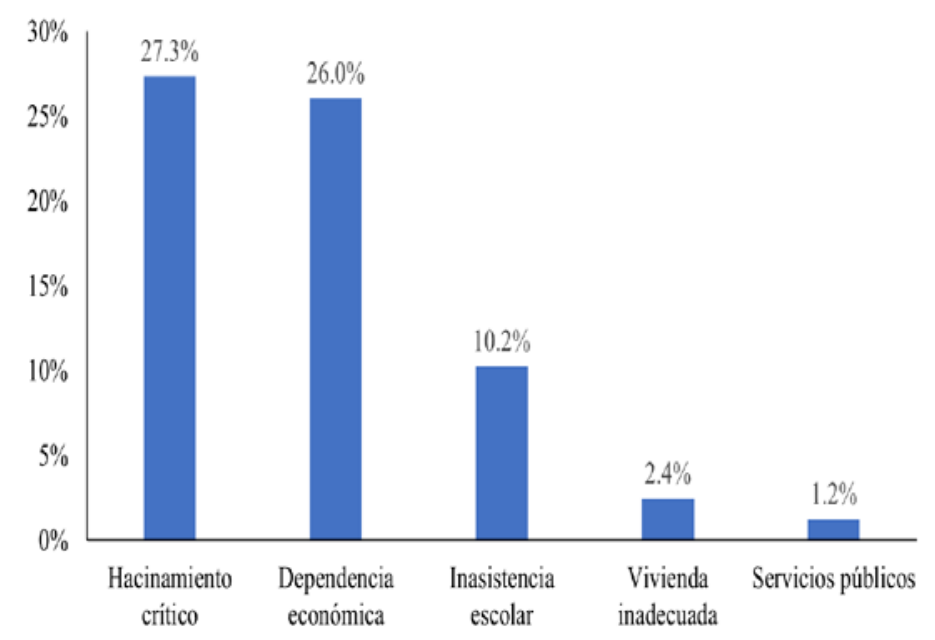

Figura 4. Necesidades básicas insatisfechas
La dependencia económica es un indicador que se construye con base en el nivel de ingreso, siendo incluidas en esta categoría las viviendas en las que hay más de tres personas por cada miembro ocupado, siempre y cuando el jefe haya alcanzado como máximo dos años de educación primaria. En ese sentido, la población con necesidades básicas insatisfechas por dependencia económica es del $26 \%$, dato que se comporta de un modo muy similar a las ocupaciones pues solo el $19.2 \%$ de los pacientes trabaja, por tanto, es una variable más que acentúa su situación de pobreza, en este caso particular, al tener en cuenta el acceso a recursos económicos, al nivel educativo y la cantidad de personas en una vivienda que dependen de esos ingresos. De las personas estudiadas con necesidades básicas insatisfechas, el 28.8\% está en situación de pobreza y el 17.4\% en situación de pobreza extrema, es decir, que alrededor de 2 de cada 10 pacientes viven en hogares que como mínimo presentan dos necesidades básicas insatisfechas y al ser las dos principales el hacinamiento crítico y la dependencia económica, es un aliciente para seguir resaltando que las condiciones para llevar a cabo un tratamiento o cuando menos garantizar una serie de certidumbres en la cotidianidad de los pacientes, que son necesarias para la salud mental del paciente dado el diagnóstico por esquizofrenia, trastornos esquizotípicos y trastornos de ideas delirantes, no son favorables y por el contrario pueden ser un motivo de crisis o de complicaciones en su proceso de atención. La distribución espacial de los pacientes que residen en la zona urbana de Cali permite establecer vínculos tanto con cada una de las variables que se han ido describiendo en los párrafos anteriores como con los resultados de 
Sánchez-Salcedo, J. F., Arboleda, M. A., Montes, M., Castrillón, M. C., Ríos, S. V., \& Soto, A. (2018). Perfil social y económico de los pacientes diagnosticados con esquizofrenia en Cali. Revista de Sociología 33(1), 58-76. doi: 10.535/0719-529X.2018.51850 otras investigaciones en las que son justamente las zonas en las que se ubican de modo más frecuente los pacientes, las que se caracterizan por presentar los indicadores sociodemográficos más preocupantes en términos de vulnerabilidad (Barbary \& Urrea, 2004). En los trabajos ya citados se sugiere pensar en la zona urbana de Cali en cinco categorías al agrupar barrios y comunas no solo por ser cercanos entre si en una perspectiva espacial, sino porque se asemejan en términos sociales, económicos y demográficos. Esta distribución es: Centro-Norte (comunas 3, 4, 9 y 10); Oriente (comunas 6, 13, 14, 15, 16 y
21); Norte-Sur (comunas 2, 5, 17, 19 у 22); Ladera (comunas 1, 18 y 20); y Nororiente (comunas 7, 8, 11 y 12). De esta manera en la Figura 5 se observa que es el conglomerado Oriente el que predomina, seguido por la Ladera, aunque destacando que el HDPUV se sitúa en la comuna 18 y hay que pensar en ello porque puede ser que su ubicación sea un motivo para atender más casos de personas de barrios aledaños a diferencia de lo que puede suceder con otras áreas de la ciudad desde las cuales pueden surgir dificultades para los pacientes que se deban trasladar hacia la institución.

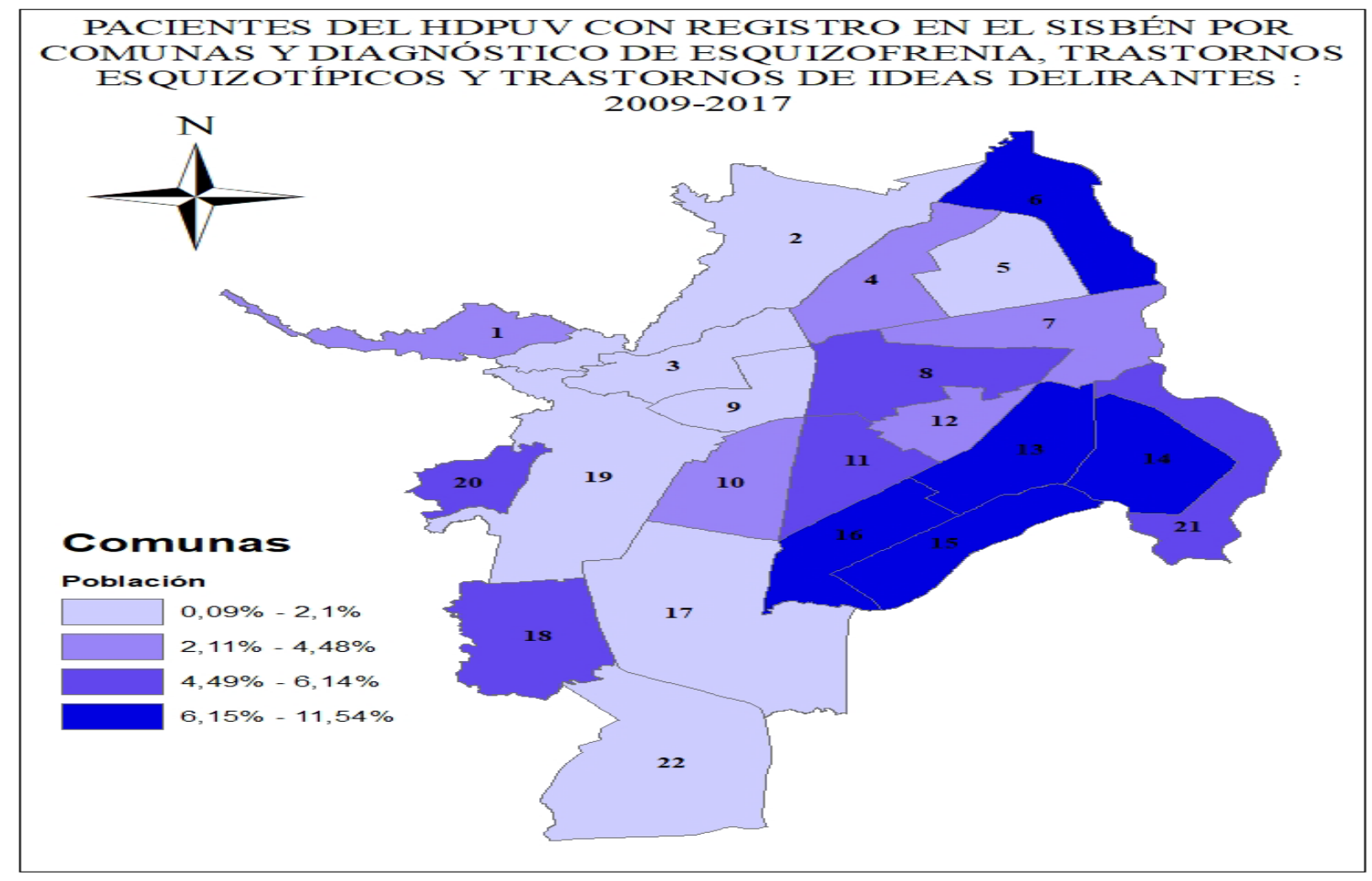

Figura 5. Distribución espacial de los pacientes por comuna

A grandes rasgos se apunta a pensar a través de los conglomerados en que existe una coincidencia entre el perfil sociodemográfico de los pacientes con algún diagnóstico del clúster de esquizofrenia, trastornos esquizotípicos y trastornos de ideas delirantes y su distribución 
espacial en términos de indicadores demográficos, sociales y económicos de acuerdo con trabajos anteriores al resaltar que son zonas de la ciudad con condiciones de vulnerabilidad muy marcadas por datos como el de un menor grado de escolaridad, acceso a la educación formal, participación en el mercado laboral, necesidades básicas insatisfechas, etc. Además, en la Encuesta Nacional de Salud Mental de 2015 los datos mostraron que la prevalencia de cualquier trastorno mental para los últimos 12 meses en los adultos que provenían de hogares vulnerables (4.9\%) y en situación de pobreza (4.4\%) fue mayor que el del promedio nacional (4\%) (MINSALUD \& COLCIENCIAS, 2015), un hallazgo que da pie a pensar en que cada una de las condiciones de pobreza y vulnerabilidad que se han descrito en los párrafos anteriores, son elementos que se pueden relacionar con la enfermedad mental en la población adulta.

Con el ánimo de matizar los datos, hay que resaltar que el HDPUV es una entidad que atiende en su mayoría a las personas que por su situación tienden a ser clasificadas dentro de las categorías de vulnerabilidad o de pobreza, mientras que otras poblaciones con mayores recursos pueden contar con la opción de recurrir a centros de atención en salud mental en otras locaciones y posibilidades de atención, como una clínica privada, por ejemplo.

Especificidades de los tipos de diagnósticos

A continuación, se muestra cómo es la distribución de los diagnósticos al interior del clúster esquizofrenia, trastornos esquizotípicos y trastornos de ideas delirantes.

Tabla 2. Diagnósticos del clúster desagregados

\begin{tabular}{|c|c|c|c|c|c|}
\hline Tipos de diagnósticos del clúster & $\mathrm{n}$ & $\%$ & $\begin{array}{l}\text { Submuestra tipos de } \\
\text { esquizofrenia }\end{array}$ & $\mathrm{n}$ & $\%$ \\
\hline Esquizofrenia & 2,612 & $76.8 \%$ & Indiferenciada & 1,998 & $76.5 \%$ \\
\hline Trastorno esquizoafectivo & 404 & $11.9 \%$ & Paranoide & 288 & $11.0 \%$ \\
\hline Trastorno psicótico agudo y transitorio & 270 & $7.9 \%$ & Sin especificación & 139 & $5.3 \%$ \\
\hline Trastorno ideas delirantes persistentes & 91 & $2.7 \%$ & Hebefrénica & 54 & $2.1 \%$ \\
\hline Psicosis no orgánica sin especificación & 17 & $0.5 \%$ & Residual & 25 & $1.0 \%$ \\
\hline Trastorno esquizotípico & 4 & $0.1 \%$ & Catatónica & 13 & $0.5 \%$ \\
\hline \multirow[t]{2}{*}{ Otros Trastorno psicóticos } & 2 & $0.1 \%$ & Simple & 12 & $0.5 \%$ \\
\hline & & & Otras esquizofrenias & 83 & $3.2 \%$ \\
\hline Total & 3,400 & $100 \%$ & & 2,612 & $100 \%$ \\
\hline
\end{tabular}

Como se aprecia en la Tabla 2, la esquizofrenia predomina dentro del clúster donde poco más de tres cuartos de la población se ubica, seguida por los trastornos esquizoafectivos y por los trastornos psicóticos agudos y transitorios. La esquizofrenia es una enfermedad que en investigación a nivel mundial es de baja prevalencia, pero como se ha descrito para el caso del HDPUV de la población con SISBÉN su diagnóstico es frecuente e incluso es de los más recurrentes en la institución, hallazgo que se explica por ser el HDPUV el principal centro de 
referencia del suroccidente colombiano para trastornos mentales graves, de manera que hay una filtración previa desde otros niveles de servicio asistencial, que posteriormente derivan a este centro monovalente de salud mental. Por sus características, la esquizofrenia y los trastornos psicóticos que se asocian, representan condiciones que incapacitan de forma grave y acarrean impactos adversos y significativos para el paciente, familia y comunidad en general. Así mismo, existen estudios como el de Thornicroft et al. (2004) en Europa, en los que se ha demostrado que las personas con trastornos mentales graves de modo usual son solteras, desempleadas, con redes de contactos nulas o limitadas, con un contacto constante con su familia y cuyas actividades recreativas son mínimas. En otras palabras, aunque los datos aportados no son un indicio suficiente para plantear una relación causal semejante, sí que hay pistas para relacionar estos fenómenos sociales y económicos con la enfermedad mental.

\section{DISCUSIÓN Y CONCLUSIONES}

En un reporte del año 2001 la Organización Mundial de la Salud (OMS, 2001) planteó que la esquizofrenia era la novena causa de años perdidos por discapacidad para la población entre los 15 a 44 años de edad. En trabajos más recientes se han presentado cambios en los últimos años en relación con la carga de la enfermedad a nivel global, donde su mortalidad decreció (Lozano et al., 2012). En ese sentido, la esquizofrenia es un trastorno mental grave y que incapacita. En la mayoría de los casos inicia en la adolescencia o adultez temprana, razón por la que una proporción considerable de pacientes no consigue desarrollarse personal o profesionalmente e incluso -en muchos casos- son incapaces de poder vivir de manera independiente o acceder al mercado laboral. Aunque a grandes rasgos los resultados de esta investigación respaldan lo anterior, lo cierto es que entre el global de diagnósticos, el clúster estudiado corresponde a casi el $20 \%$ de los casos y entre este total el $76.8 \%$ es un diagnóstico de esquizofrenia en cualquiera de sus formas, es decir, los datos son mucho más altos respecto a las cifras globales y se confirma que en efecto el
HDPUV es una institución que atiende a pacientes con trastornos mentales agudos y tal como se propuso previamente, tendría que ser analizado a la luz de las circunstancias de organización de los servicios psiquiátricos, que llevan a centralizar la atención aguda de esta población en el HDPUV, pero también lleva a preguntarse acerca de los criterios diagnósticos utilizados por los profesionales de la institución, de manera que se descarte un posible sesgo que posibilite el sobre diagnóstico de este clúster.

Se encuentra que la mayor parte de la población sí está afiliada al régimen de aseguramiento en salud, pero el $31.5 \%$ de quienes no lo están sigue siendo una cifra a destacar, porque es un grupo de pacientes desprotegidos, que en este caso particular no sólo deben enfrentarse a unas condiciones de un alto grado de dificultad en un sentido económico, social o de salud, sino por el tipo de diagnóstico que se les registra y por ende las vías de atención a seguir.

Lo anterior es un indicio de cómo los pacientes en esta situación se enfrentan a un ciclo que pareciera no tener fin, ya que su condición de vulnerabilidad y los riesgos de una agudización 
de su enfermedad mental se mantienen o acentúan. En otras palabras, se torna complejo que ese círculo vicioso se pueda romper. A su vez, no hay que pasar por alto que la enfermedad mental está rodeada por prejuicios, comportamientos discriminatorios y estigmas. Con frecuencia, una persona diagnosticada con una enfermedad mental, se enfrentan a contextos en los que se restringen sus derechos legales, experimentan inequidades en su proceso de atención o son devaluados socialmente. Además, tanto las situaciones descritas como la enfermedad mental en sí misma, se van infiltrando en la mente de la persona diagnosticada y esto acarrea una baja en la autoestima, negarse a buscar un tratamiento o dificultar aún más su tratamiento (Boysen et al., 2014).

Desde una mirada institucional los costos de manejo y tratamiento de la enfermedad mental son altos y esto puede ocasionar que la atención en ciertas ocasiones sea imposible de brindar para los pacientes. $\mathrm{Al}$ respecto se resalta que son varias las aproximaciones terapéuticas que pueden actuar de modo complementario como el tratamiento farmacológico, tratamiento psicosocial y psicoterapéutico, sin pasar por alto la diversificación en las modalidades de atención al paciente. Por tanto, un trastorno mental como la esquizofrenia al afectar diferentes áreas de la salud y la vida de la persona, precisa de un manejo integral de su condición. Finalmente, pese a la idea anterior la evidencia ha demostrado que la mayor parte de los pacientes con este diagnóstico se les ofrece únicamente un tratamiento psicofarmacológico (Falloon et al., 2004).

En relación con las redes de apoyo, Tejada (2016) en uno de los apartados de su documento habla sobre la "situación del cuidador" en torno a las variables vinculadas con la enfermedad del paciente, pero que terminan por impactar la calidad de vida de la o las personas que le cuidan. Sobre ello menciona que pese a ser una tarea gratificante para familiares o personas cercanas, los cuidadores en buena parte de los casos se enfrentan a cantidades significativas de estrés o inconvenientes a nivel físico, social y económico, con un escaso apoyo de los profesionales en salud mental. Así mismo, se muestra que en los países en desarrollo son las familias las que suelen ser una red clave del cuidado, siendo las mujeres las que están a cargo (aunque los hombres asumen estas labores cada vez más). De este modo, se comprende que las condiciones de vulnerabilidad descritas en el artículo no sólo afectan de manera directa la calidad de vida del paciente o a las instituciones y profesionales a cargo de su proceso de atención, sino a una red cercana al paciente que en la mayor parte de los casos son sus familiares.

Aunque no creemos posible establecer una relación causal entre pobreza y enfermedad mental, lo que sí se identifica es una coincidencia entre la distribución espacial de los pacientes, los resultados de investigaciones similares que integran entre sus objetivos variables a nivel social, económico y demográfico, y los indicadores del perfil sociodemográfico que se han descrito en este documento. Es decir, surgen nuevas posibilidades de estudio en las que se relacione la salud mental con variables como la pobreza monetaria, ocupación, hacinamiento, necesidades básicas insatisfechas, nivel de ingreso, estrato, etc.

Finalmente, con una definición del problema como se ha propuesto en este artículo y las opciones para futuras investigaciones, se 
Sánchez-Salcedo, J. F., Arboleda, M. A., Montes, M., Castrillón, M. C., Ríos, S. V., \& Soto, A. (2018). Perfil social y económico de los pacientes diagnosticados con esquizofrenia en Cali. Revista de Sociología 33(1), 58-76. doi: 10.535/0719-529X.2018.51850 demuestra que hay una necesidad por ahondar en esta clase de asuntos desde los cuales se busca promover una mirada más incluyente y abierta a la comprensión de la salud mental, sus pacientes, tratamientos y atención. Por supuesto, es necesario entonces que se conciba la enfermedad mental de modo distinto y se redireccionen las estrategias de salud pública para expandir y complejizar su marco de análisis, comprensión y atención.

\section{REFERENCIAS}

Alemán, A., Kahn, R. S., \& Selten, J.-P. (2003) Sex differences in the risk of schizophrenia. Evidence from meta-analysis. Archives of General Psychiatry, 60(6), 565-571. doi: 10.1001/archpsyc.60.6.565

Barbary, O., \& Urrea, F. (2004). Gente negra en Colombia: Dinámicas sociopolíticas en Cali y el Pacífico. Medellín, Colombia: Editorial Lealon.

Boysen, G., Ebersole, A., Casner, R., \& Coston, N. (2014). Gendered mental disorders: Masculine and feminine stereotypes about mental disorders and their relation to stigma. The Journal of Social Psychology, 154(6) 546-565. doi: 10.1080/00224545.2014.953028

Carr, V. J., Neil, A. L., Halpin, S. A., Holmes, S., \& Lewin, T. J. (2003). Costs of schizophrenia and other psychoses in urban Australia: Findings from the Low Prevalence (Psychotic) Disorders Study. Australian and New Zealand Journal of Psychiatry, 37(1), 31-40. doi: $\quad 10.1046 / j .1440-$ 1614.2003.01092.x

Castillo-Martell, H. (2014) Impacto de la esquizofrenia en la calidad de vida y desarrollo humano de pacientes y familias de una institución de atención en salud mental de la ciudad de Lima. Anales de Salud Mental, 30(2), 9-27.

Departamento Administrativo Nacional de Estadística. (2017). Pobreza monetaria y multidimensional en Colombia. Recuperado de

http://www.dane.gov.co/files/investigaciones /condiciones_vida/pobreza/bol_pobreza_16.p df

Falloon, I. R., Montero, I., Sungur, M., Mastroeni, A., Malm, U., Economou, M., ... Gedye, R. (2004). Implementation of evidence-based treatment for schizophrenic disorders: Two-year outcome of an international field trial of optimal treatment. World Psychiatry, 3(2), 104-109.

Ministerio de Salud y Protección Social, \& Departamento Administrativo de Ciencia, Tecnología e Innovación (2015). Encuesta Nacional de Salud Mental 2015. Bogotá, Colombia: Javegraf

Hopper, K. J., \& Wanderling, J. (2000). Revisiting the developed versus developing country distinction in course and outcome in schizophrenia: results from ISoS, the WHO collaborative followup project. International Study of Schizophrenia. Schizophrenia Bulletin, 26(4), 835-846. doi: 10.1093/oxfordjournals.schbul.a033498 
Sánchez-Salcedo, J. F., Arboleda, M. A., Montes, M., Castrillón, M. C., Ríos, S. V., \& Soto, A. (2018). Perfil social y económico de los pacientes diagnosticados con esquizofrenia en Cali. Revista de Sociología 33(1), 58-76. doi: 10.535/0719-529X.2018.51850
Jablensky, A. (2000). Epidemiology of schizophrenia: The global burden of disease and disability. European Archives of Psychiatry and Clinical Neuroscience, 250(6), 274-285. doi: $10.1007 / \mathrm{s} 004060070002$

León, C. A., León, A. (1996) Curso clínico y evolución de la esquizofrenia en Cali. Un estudio de seguimiento de 26 años. Salud Mental, 19(2), 12-35.

Lieberman, J. A., \& First, M. B. (2018). Psychotic disorders. The New England Journal of Medicine, $379(1), \quad 270-280 . \quad$ doi: 10.1056/NEJMra1801490

Lozano, R., Naghavi, M., Foreman, K., Lim, S., Shibuya, K., Aboyans, V., ... Murray, C. J. L. (2012). Global and regional mortality from 235 causes of death for 20 age groups in 1990 and 2010: A systematic analysis for the Global Burden of Disease Study 2010. The Lancet, $\quad 380(9859), \quad 2095-2128 . \quad$ doi: 10.1016/S0140-6736(12)61728-0

McGrath, J., Saha, S., Welham, J., El Saadi1, O., MacCauley, C., \& Chant, D. (2004). A systematic review of the incidence of schizophrenia: The distribution of rates and the influence of sex, urbanicity, migrant status and methodology. BMC Medicine, 2(13), 1-22. doi: 10.1186/1741-7015-2-13

McGrath, J., Saha, S., Chant, D., \& Welham, J. (2008). Schizophrenia: A concise overview of incidence, prevalence, and mortality. Epidemiologic Reviews, 30(1), 67-76. doi: 10.1093/epirev/mxn001

Organización Mundial de la Salud (2001). Informe sobre la salud en el mundo 2001 Salud mental: Nuevos conocimientos, nuevas esperanzas. París, Francia: Organización Mundial de la Salud.
Read, J. (2010). Can poverty drive you mad? 'Schizophrenia', socio-economic status and the case for primary prevention. New Zealand Journal of Psychology, 39(2), 7-19.

Saha, S., Chant, D., Welham, J., \& McGrath, J. (2005). A systematic review of the prevalence of schizophrenia. PLoS Medicine, 2(5), 413-433. doi: 10.1371/journal.pmed.0020141

Salokangas, R. K., Honkonen, T., Stengard, E., Koivisto, A. M. (2001). To be or not to be married - that is the question of quality of life in men with schizophrenia. Social Psychiatry and Psychiatric Epidemiology, 36(8), 381-390. doi: 10.1007/s001270170028

Sánchez, R., Tellez, G., Jaramillo, L. E. (2012) Edad de inicio de los síntomas y sexo en pacientes con trastorno del espectro esquizofrénico. Biomédica, 32(2), 206-213. doi: 10.7705/biomedica.v32i2.423

Tejada, P. (2016). Situación actual de los trastornos mentales en Colombia y en el mundo: Prevalencia, consecuencias y necesidades de intervención. Revista Salud Bosque, 6(2), 29-40.

Thornicroft, G., Tansella, M., Becker, T., Knapp, M., Leese, M., Schene, A., \& VazquezBarquero, J. L. (2004). The personal impact of schizophrenia in Europe. Schizophrenia Research, 69(2-3), 125-132. doi: 10.1016/S0920-9964(03)00191-9

World Health Organization. (2008). Mental Health Gap Action Programme - Scaling up care for mental, neurological, and substance use disorders. Geneva Switzerland: World Health Organization,

Manuscrito recibido: 06-09-2018 Manuscrito aceptado: 29-11-2018 